\title{
Downregulation of glutathione peroxidase 3 is associated with lymph node metastasis and prognosis in cervical cancer
}

\author{
XIANGLAN ZHANG ${ }^{1,6}$, ZHENLONG ZHENG ${ }^{3,7}$, SHEN YINGJI $^{5}$, HYEYEON KIM ${ }^{4}$, RENSHUN JIN ${ }^{1}$, \\ LI RENSHU $^{8}$, DOO YOUNG LEE ${ }^{6}$, MI RYUNG ROH ${ }^{7}$ and SANGHWA YANG ${ }^{2,9}$ \\ ${ }^{1}$ Department of Pathology, Yanbian University Hospital, Yanji, Jilin, P.R. China; ${ }^{2}$ National Creative Research Initiatives \\ Center for Inflammatory Response Modulation, Yonsei University, Seoul, Republic of Korea; ${ }^{3}$ Department of Dermatology, \\ Yanbian University Hospital, Yanji, Jilin, P.R. China; ${ }^{4}$ Department of Gynecology, Gangnam Severance Hospital, \\ Yonsei University College of Medicine, Seoul, Republic of Korea; ${ }^{5}$ Department of Gynecology, Maternal and Child Health \\ Hospital, Dalian, Liaoning, P.R. China; ${ }^{6}$ Oral Cancer Research Institute, College of Dentistry, Yonsei University, Seoul, \\ Republic of Korea; ${ }^{7}$ Department of Dermatology, Yonsei University College of Medicine, Seoul, Republic of Korea; \\ ${ }^{8}$ Department of Anesthesia and Pain, Yanbian University Hospital, Yanji, Jilin, P.R. China; \\ ${ }^{9}$ Goodgene Inc., Seoul, Republic of Korea
}

Received February 25, 2014; Accepted March 21, 2014

DOI: $10.3892 /$ or.2014.3152

\begin{abstract}
Glutathione peroxidase 3 (GPX3) is a member of the glutathione peroxidase family of selenoproteins and is one of the key defensive enzymes against oxidative damages to host cells. Downregulation of GPX3 due to its promoter hypermethylation has been documented in several different types of cancer, indicating that GPX3 functions as a possible tumor suppressor. In the present study, we showed that GPX3 is also significantly downregulated in cervical cancer tissues compared to normal cervical tissues by qRT-PCR analyses and immunohistostainings. GPX3 expression was significantly related to lymph node metastasis and prognosis in cervical cancer patients. Treatment of cervical cancer cells with 5-aza-2'-deoxycytidine restored the expression of GPX3 and methylation-specific PCR (MSP) confirmed the CpG methylation of the GPX3 gene. Our results indicate that promoter methylation is one of the major causes of GPX3 downregulation in cervical cancer and GPX 3 could serve as a predictive biomarker for lymph node metastasis and prognosis of cervical cancer.
\end{abstract}

Correspondence to: Dr Mi Ryung Roh, Department of Dermatology, Yonsei University College of Medicine, Seoul, Republic of Korea

E-mail: karenroh@yuhs.ac

Dr Sanghwa Yang, National Creative Research Initiatives Center for Inflammatory Response Modulation, Yonsei University, Seoul, Republic of Korea

E-mail: yshi@yonsei.ac.kr

Key words: cervical cancer, GPX3, downregulation, lymph node metastasis, prognosis

\section{Introduction}

Glutathione peroxidase 3 (GPX3 or plasma GPX) is a member of the glutathione peroxidase family of selenoproteins and is one of the key defensive enzymes against oxidative damages to host cells (1). A considerable amount of research has been performed on the role of glutathione peroxidases on cancer progression. Since the major biochemical role of a hydroperoxide is to regulate the characteristics of cancer cells including proliferation, invasion, migration, angiogenesis and apoptosis, GPX3 is expected to regulate cancer progression by regulating the level of hydroperoxides inside cells (2). More is now known about the relationship between the GPX3 expression, methylation and cancer progression. For example, the GPX3 gene shows a pattern of deletion or promoter hypermethylation, which is accompanied by gene silencing in multiple types of cancer, including prostate, gastric and Barrett's esophageal cancer, and conversely the overexpression of GPX3 inhibits tumor growth and metastasis (3-7). GPX3 has also been shown to be responsible for the cisplatin sensitivity of clear cell adenocarcinoma in ovarian cancer (8), and its negative expression is significantly correlated with poor prognosis of gallbladder cancer, multiple myeloma and gastric cancer $(7,9,10)$. In particular, silencing of GPX3 increases ROS production in colon cancer cell lines and muscle stem cells $(11,12)$. Another possible link between cancer suppression and GPX3, although it remains to be experimentally confirmed, is that GPX3 is a selenoprotein, an enzyme containing a selenocysteine ( $\mathrm{Sec}$ ) residue at its active site, and uptake of selenium has a potential to reduce certain cancers $(13,14)$. Human selenoproteins encode 25 genes, including glutathione peroxidases, and are all involved in crucial biological defense systems such as antioxidant defense (14). On the other hand, there are quite different patterns of gene expression among GPXs. GPX2, for example, shows elevated levels of expression, while GPX1, 3 and 4 show 
decreased expression in multiple types of cancer (2). A recent study also predicted a causal relationship between changes in GPX3 expression and in obesity-related traits (15).

Cervical cancer is the second most common cancer in women and identification and establishment of molecular markers for cervical cancer is highly necessary for improved diagnoses and development of therapeutic targets (16).

Based on the information that GPX3 is downregulated in multiple types of cancer and functions as a tumor suppressor, in the present study, we examined whether GPX3 is also downregulated in cervical cancer and, if so, whether promoter methylation is related to its repression. We investigated further the clinicopathological significance of GPX3 downregulation in cervical cancer and demonstrated the prognostic value of GPX3 in cervical cancer patients.

\section{Materials and methods}

RNA extraction and SYBR-Green real-time PCR. Total RNAs were extracted from 4 cervical cancer cell lines, 6 normal cervical tissues and 10 cervical cancer tissues using TRIzol (Invitrogen) and RNeasy Mini kit (Qiagen GmbH, Hilden, Germany). One microgram each of the quantitated total RNA was converted into cDNAs using Transcriptor High Fidelity cDNA Synthesis kit (Roche Applied Science, Netherlands) following the manufacturer's protocol. Quantitative real-time PCR was performed using 1X SYBR-Green Master Mix (Applied Biosystems), 10 pmol of each primer and $2 \mu \mathrm{l}$ of the cDNA in an Mx3005P QPCR System (Agilent Technologies, Santa Clara, CA, USA) under the following conditions: initial denaturation for $10 \mathrm{~min}$ at $95^{\circ} \mathrm{C}$, followed by 40 cycles of $95^{\circ} \mathrm{C}$ for $20 \mathrm{sec}, 50^{\circ} \mathrm{C}$ for $30 \mathrm{sec}$ and $72^{\circ} \mathrm{C}$ for $45 \mathrm{sec}$. GPX3 mRNA expression was also detected by conventional RT-PCR using Glod Taq Polymerase (Applied Biosystems) with an annealing temperature of $58^{\circ} \mathrm{C}$. The GPX3 expression was normalized by the expression of $\beta$-actin. Oligonucleotide primers used for the PCR were: '5-CAACCAATTTGGAAAACAGG-3' and 5'-GTGGGAGGACAGGAGTTCTT-3' for GPX3; and 5'-ATA GCACAGCCTGGATAGCAACGTAC-3' and 5'-CACCTTCT ACAATGAGCTGCGTGTG-3' for $\beta$-actin.

Cell culture and 5-Aza-2'-deoxycytidine (5-Aza-dC) treatment. Cervical cancer cell lines (HeLa, SiHa, CaSki and ME-180) were purchased from the Korean Cell Line Bank (Seoul, Korea). Cells were maintained in minimum essential medium (SigmaAldrich, St. Louis, MO, USA) supplemented with fetal bovine serum to a final concentration of $10 \%$, streptomycin (final concentration, $100 \mu \mathrm{g} / \mathrm{ml}$ ) and penicillin (final concentration, $50 \mathrm{U} / \mathrm{ml}$ ) at $37^{\circ} \mathrm{C}$ in a humidified incubator containing $5 \% \mathrm{CO}_{2}$. For the demethylation experiment, $5 \times 10^{5}$ cells were grown for $24 \mathrm{~h}$ prior to 5-Aza-dC (Sigma-Aldrich) treatment. 5-Aza-dC in PBS was filtered and treated to the final concentration of $1 \mu \mathrm{M}$ for 3 days, with fresh 5-Aza-dC changes at every $24 \mathrm{~h}$. PBS-treated cells were grown alongside as untreated controls.

DNA extraction and methylation-specific PCR (MSP). Genomic DNA was extracted from 4 cervical cancer cell lines (HeLa, SiHa, CaSki and ME-180) using QIAamp DNA mini kit (Qiagen GmbH, Hilden, Germany). MSP was based on a previous report (17). Genomic DNA (500 ng) was used for
Table I. Clinicopathological characteristics of cervical squamous cell carcinoma patients.

\begin{tabular}{lc}
\hline Variables & No. of patients $(\%)$ \\
\hline Total cases & 50 \\
Age (years) & \\
Median age (range) & $53(32-70)$ \\
$<53$ & $24(48.0)$ \\
$\geq 53$ & $26(52.0)$ \\
Tumor depth & \\
Tis & $3(6.0)$ \\
T1 & $42(84.0)$ \\
T2 & $5(10.0)$ \\
LN metastasis & \\
N0 & $31(62.0)$ \\
N1 & $19(38.0)$ \\
Stage & \\
0 & $3(6.0)$ \\
I & $27(54.0)$ \\
II & $1(2.0)$ \\
III & $19(38.0)$ \\
\hline
\end{tabular}

bisulfite conversion overnight following a protocol included in the EZ DNA Methylation kit (Zymo Research Corporation, Orange, CA, USA). Oligonucleotide primers used for amplifying the $\mathrm{CpG}$ islands for GPX3 promoter regions were: 5'-TATGTTATTGTCGTTTCGGGAC-3' and 5'-GTCCGT CTAAAATATCCGACG-3' for methylation-specific amplification; and 5'-TTTATGTTATTGTTTTGGGATG-3' and 5'-ATCCATCTAAAATATCCAACACTCC-3' for non-methylation-specific amplification. Amplification was performed in a cycle of $95^{\circ} \mathrm{C}$ for $10 \mathrm{~min}, 40$ cycles of $94^{\circ} \mathrm{C}$ for $30 \mathrm{sec}, 59^{\circ} \mathrm{C}$ for $30 \mathrm{sec}$ and $72^{\circ} \mathrm{C}$ for $30 \mathrm{sec}$, followed by the final incubation at $72^{\circ} \mathrm{C}$ for $10 \mathrm{~min}$. The amplified DNA products were resolved with electrophoresis on $1 \%$ agarose gels.

Public microarray data acquisition and analysis. Gene expression data related to cervical cancer were downloaded from either Gene Expression Omnibus (www.ncbi.nlm.nih.gov/geo) or Array Express (www.ebi.ac.uk/arrayexpress). Gene expression profiling data containing cervical cancer cell lines, normal cervices and cervical cancer tissues (GSE 9750) and non-malignant cervical tissues and various FIGO stages of carcinomas (GSE 46857) were used to test the differences in GPX3 mRNA expression levels. A methylation profiling experiment performed on Illumina Human Methylation 27 BeadChip (GSE 30760) was used to probe methylation level of CpG islands of GPX3 in normal cervices and cervical tumor tissues. Microarray analyses were performed using BRB-Array Tools developed by Dr Richard Simon and BRB-Array Tools Development Team or using GeneSpring 12.6.

Immunostaining of GPX3 on surgical specimens. The present study included 50 cervical cancer patients who were 
A

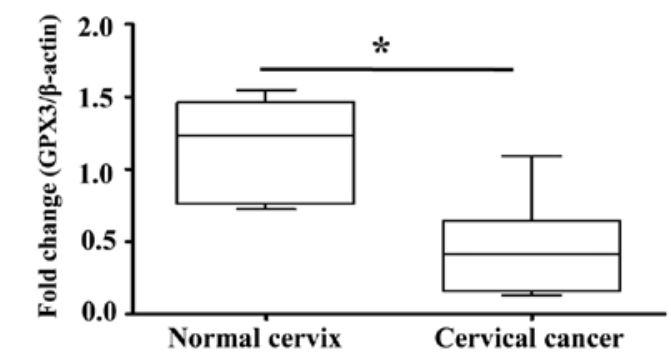

C

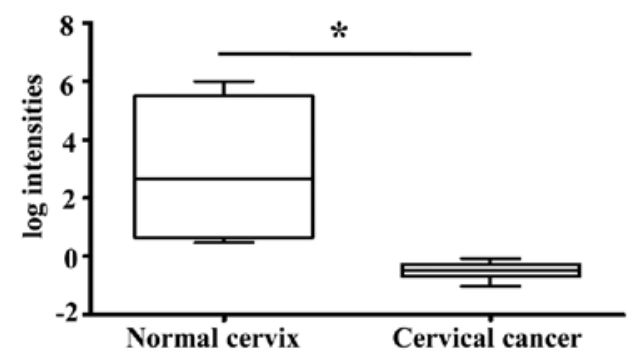

B

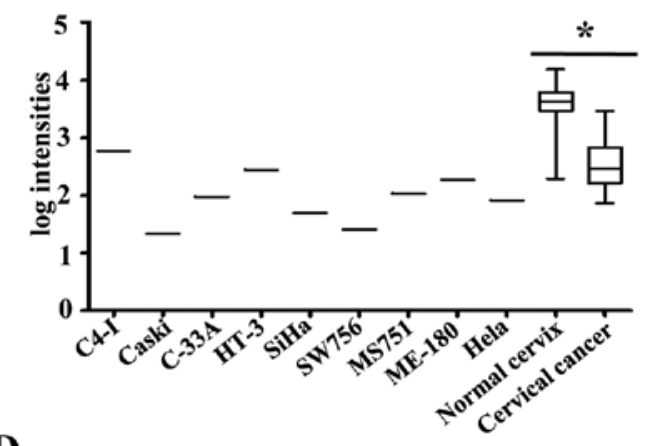

D

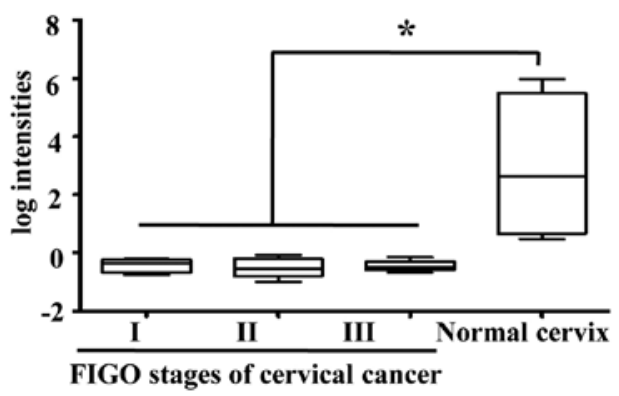

Figure 1. GPX3 mRNA expression in cervical cancer cell lines and surgical tissue samples. (A) GPX3 mRNA expression was significantly decreased in cervical cancer tissues compared to normal cervix $(\mathrm{p}<0.001)$. $\beta$-actin was used as an internal control in all measurements. (B) In a gene expression profiling of cervical cancer cell lines, cervical cancer tissues and normal cervical tissues, performed on an Affymetrix Human Genome U133A Array format (GSE 9750), GPX3 showed $~ 11$ and 35-fold downregulation in cervical tumor tissues and cancer cell lines, respectively, compared with GPX3 expression in normal cervix tissues $(\mathrm{p}<0.001)$. (C) In a global gene expression profiling study that compared normal cervical tissues and various FIGO stages of cervical cancer tissues (GSE 46857), GPX3 showed an average of 11-fold downregulation in cervical cancer tissues compared with normal cervical tissues ( $<<0.001$ ). (D) However, when the cancer samples in Fig. 2C were analyzed in detail, there were no differences in expression levels of GPX3 among cancer tissues obtained from patients with different FIGO stages. Data in Fig. $2 \mathrm{C}$ and D are from a common dataset, the only difference being the cancer samples are shown in detailed FIGO groups in Fig. 2D. GPX3, glutathione peroxidase 3.

diagnosed with cervical cancer in Yonsei University Hospital, Korea, between 2000 and 2004. Clinicopathological characteristics of the patients are shown in Table I. The present study was approved by the Institutional Review Board of Yonsei University Hospital. Formalin-fixed, paraffin-embedded surgical specimens were cut into $4 \mu \mathrm{M}$ tissue sections and deparaffinized with xylene. After hydrating with graded ethanol, endogenous peroxidase activity was blocked with a mixture of methanol and $\mathrm{H}_{2} \mathrm{O}_{2}$ at a ratio of 40:1. Antigen retrieval was performed with antigen retrieval buffer (Dako, Carpinteria, CA, USA) and primary antibody incubation was performed at RT for $1 \mathrm{~h}$ (mouse monoclonal anti-human GPX3 antibody, working dilution; 1:100) (Abcam, Cambridge, MA, USA). Real ${ }^{\mathrm{TM}}$ EnVision $^{\mathrm{TM}}$ HRP rabbit/mouse detection system (Dako) was used as secondary antibody. The sections were developed with 3,3'-diaminobenzidine (DAB) chromogen and counterstaining was carried out with hematoxylin. For analysis, patients were divided into two groups based on the expression level of GPX3; negative (no or $<5 \%$ positive cells) and positive ( $>5 \%$ positive cells) group. The survival estimates were based on Kaplan-Meier survival curves along with 95\% CI and the log-rank test was used to compare survival curves. A chi-square test was used to analyze the relationship between GPX3 protein expression and various clinicopathological parameters. Fisher's exact test was used to determine the difference between matched normal and cancer tissues in GPX3 expression.

\section{Results}

GPX3 is downregulated in cervical cancer. The mRNA expression of GPX3 was comparatively investigated in 6 normal cervix and 10 cervical cancer tissues. We found that GPX3 mRNA expression was significantly decreased in cervical cancer tissues compared to normal cervical tissues (Fig. 1A). We also examined the GPX3 expression profiles in other published genomic data. In a set of public microarray data containing normal cervices, cervical cancer tissues and cell lines (18), GPX3 showed significant downregulation in cervical cancer tissues and cell lines compared to normal cervical tissues (Fig. 1B). In other independent microarray data, GPX3 expression was again significantly repressed in cervical cancer compared to normal cervical tissues (Fig. 1C). However, there were no differences in expression levels of GPX3 among cancer tissues obtained from patients with different FIGO stages (Fig. 1D). Collectively, these results suggest that GPX3 is significantly downregulated in cervical cancer compared to its normal counterpart.

In silico analysis of methylation profile of GPX3 in cervical cancer. DNA methylation is one of the most prominent epigenetic mechanisms that control gene expressions and, therefore, disease progression (19-21). Previous results showed GPX3 downregulation in gastric cancer, Barrett's adenocarcinomas and prostate cancer and their concurrent promoter hyper- 
A

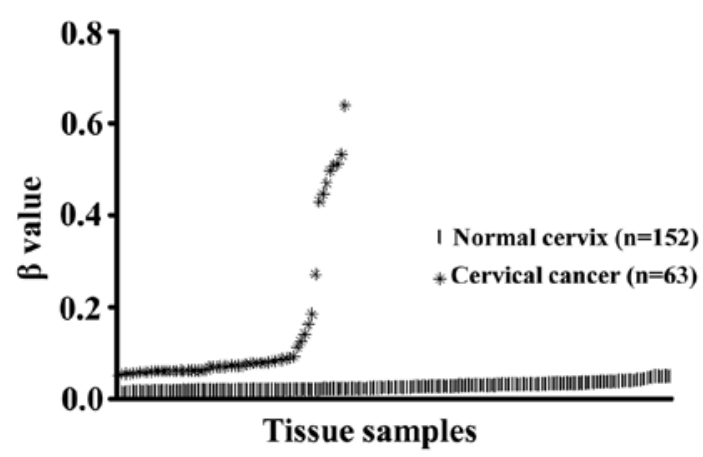

B

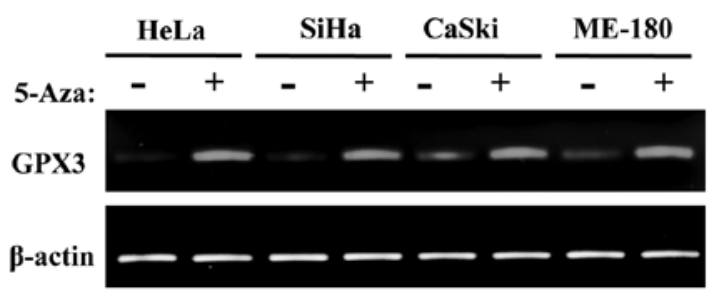

C

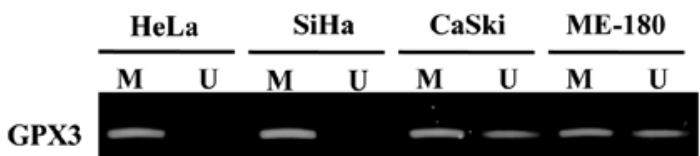

Figure 2. CpG hypermethylation is partly responsible for the downregulation of GPX3 in cervical cancer cells. (A) In silico analysis of GPX3 promoter methylation. Methylation profiling data, performed on Illumina Human Methylation 27 BeadChip (GSE 30760), were downloaded (as described in Materials and methods) and $\beta$ values for GPX3 were analyzed. The $\beta$ value represents the proportion of methylated signal intensities among all intensities (methylated and unmethylated intensities) in each probe, and it is always a number between 0 and 1 , with 0 being non-methylation among CpG islands analyzed and 1 representing full methylation. The mean, median and standard deviations were $0.027,0.025$ and 0.009 for normal cervical tissues; and $0.1327,0.072$ and 0.148 for cervical cancer tissues, respectively. (B) RT-PCR analysis in cervical cancer cell lines with or without 5-Aza-dC (1 $\mu \mathrm{M})$ treatment; restoration of GPX3 mRNA expression was found in all cervical cancer cell lines with 5-Aza-dC $(1 \mu \mathrm{M})$ treatment. (C) MSP analysis in cervical cancer cell lines. Full methylations for GPX3 were found in HeLa and SiHa cell lines and partial methylations were found in CaSki and ME-180 cell lines. GPX3, glutathione peroxidase 3; 5-Aza-dC, 5-Aza-2'-deoxycytidine.

A $\mathbf{i}$

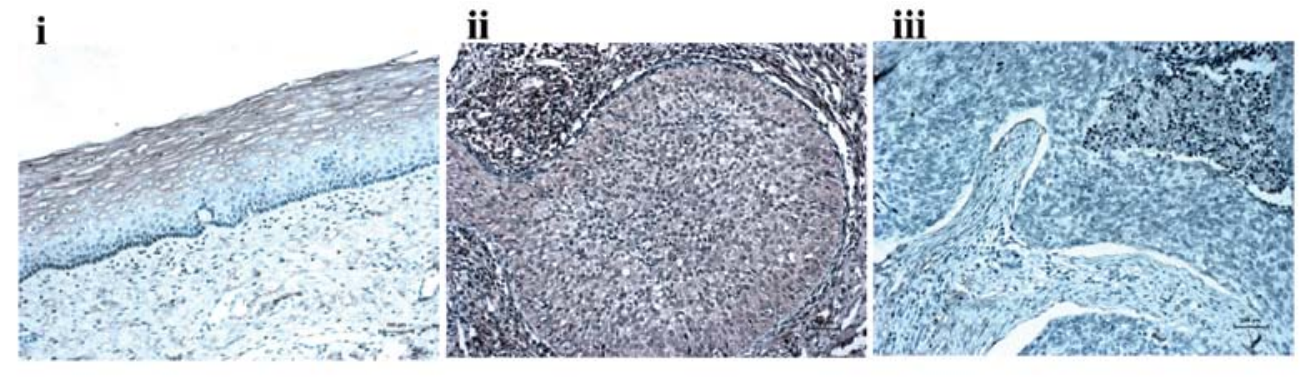

B

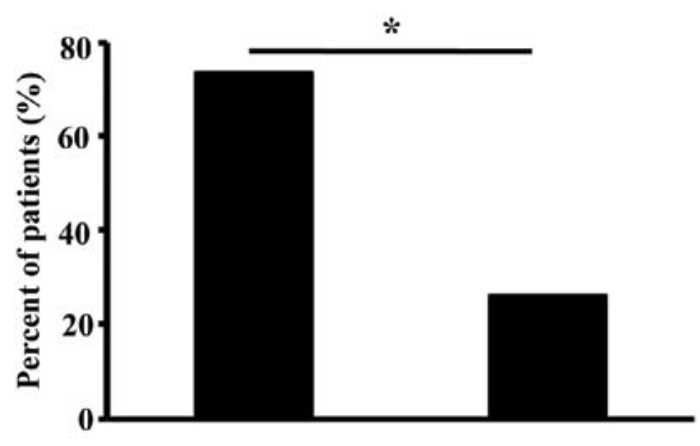

C

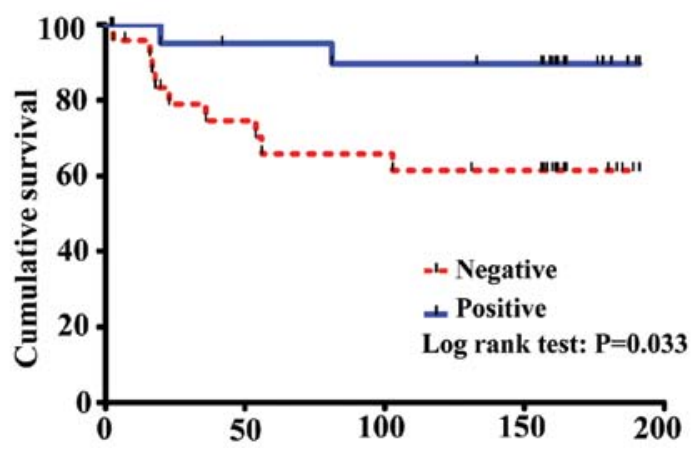

Figure 3. Protein expression of GPX3 in cervical cancer patients. (A) GPX3 expression in (i) normal cervical tissues and (ii and iii) cervical cancer tissues. (B) Protein expression of GPX3 was associated with lymph node metastasis in cervical cancer patients. Lymph node metastasis was more frequently found in patients without GPX3 expression than in patients with GPX3 expression ( $p=0.049)$. (C) Survival analysis; GPX3 expression was significantly associated with overall survival in cervical cancer patients $(\mathrm{p}=0.033)$. GPX3, glutathione peroxidase 3 .

methylation (1,3,5-7). We searched the epigenome analysis database of normal and cancer tissues from the uterine cervix to examine the differential methylation degrees of GPX3. In a methylation profiling study comparing 152 normal uterine cervical tissues and 63 cervical cancer tissues (GSE 30760), GPX3 showed $\beta$ values close to zero (no methylation in known $\mathrm{CpG}$ islands) in almost all normal tissues, whereas there was a significant trend of increase in $\beta$ values in tumor samples, indicating an elevated degree of methylation in $\mathrm{CpG}$ islands of GPX3 (Fig. 2A). This is consistent with previous reports that GPX3 repression in cancer is due to hypermethylation or deletion. This finding shows that there is a higher degree of $\mathrm{CpG}$ methylation in GPX3 promoter region in cervical cancer tissues compared to normal cervical tissues. 
Reversion of GPX3 downregulation by a demethylating agent in cervical cancer cells. To directly test whether GPX3 repression in cervical cancer is correlated with promoter methylation, we compared the GPX3 mRNA expression levels in cervical cancer cells with or without 5-Aza-dC treatment, a potent demethylating agent (22). In HeLa and SiHa, significant upregulation of GPX3 mRNAs, following a 5-Aza-dC treatment, were observed, whereas in CaSki and ME-180, relatively mild but still clear upregulation of GPX3 mRNAs were evident in the same conditions (Fig. 2B). To directly test the promoter methylation, bisulfite-converted genomic DNAs from the same cervical cancer cells were subjected to MSP. In HeLa and $\mathrm{SiHa}$, only methylation-specific amplified DNA fragments were visible, indicating full methylation of $\mathrm{CpG}$ islands, whereas in CaSki and ME-180, both of the methylation-specific and unmethylation-specific amplified DNA fragments were visible, indicating partial methylation of $\mathrm{CpG}$ islands in these two cell lines. The degree and pattern of $\mathrm{CpG}$ methylation in different cervical cancer cell lines are consistent with those of upregulation of GPX3 following a 5-Aza-dC treatment. Therefore, GPX3 is downregulated in cervical cancer due to CpG hypermethylation, although methylation may not be the only mechanism suppressing its transcription.

Immunostaining for GPX3 in normal cervix and cervical cancer tissues. GPX3 was mainly expressed in cytoplasm of the cells, and its expression was significantly increased in normal cervix $(6 / 6,100 \%)$ compared to cervical cancer tissues (22/50, 44.0\%, p=0.023; Fig. 3A). Furthermore, lymph node metastases were observed more often in patients without GPX3 expression $(73.7 \%$ ) than in patients with GPX3 expression (26.3\%, p=0.049; Fig. 3B). Patients without GPX3 expression also showed poorer prognosis than patients with GPX3 expression $(p=0.033$, median survival duration was 117 months for low GPX3 vs. 160.5 months for high GPX3; Fig. 3C).

\section{Discussion}

In humans, there are 5 selenocysteine-containing glutathione peroxidases (GPX1-4 and GPX6), and they all promote the reduction of hydroperoxides by means of glutathione (2 glutathiones $+\mathrm{H}_{2} \mathrm{O}_{2} \leftrightarrow$ glutathione disulfide $+2 \mathrm{H}_{2} \mathrm{O}$ ), thereby acting as antioxidant enzymes that remove hydroperoxides (23). GPX3, in particular, is known to function as a transcription factor in the form of a homotetramer. Selenium uptake is correlated with decreased cancer incidence and the chemopreventive function is evident especially to those with low selenium level $(23,24)$. The relationship between the selenium level and cancer development, GPX3 being the selenoprotein and downregulated in certain types of cancer, might suggest that the in vivo selenium level determines the tumor suppressor activity of GPX3, which functions to regulate the in vivo hydroperoxide level and therefore oxidative damages to the cancer cell. However, to date, no direct experimental evidence for these links has been provided (2).

Epigenetic modulation of gene expression is a significant complement to other genetic alterations including gene amplifications and deletions, where a significant amount of DNA sequence changes are accompanied. Several reports have detailed the relationship between GPX3 expression and its correlation with cancer progression (25). For example, in prostate cancer, GPX3 is methylated at its promoter in $93 \%$ of the cancer tissues examined and its mRNA expression is concurrently repressed, whereas robust mRNA expression was observed in normal prostate epithelial cells (1). In the present study, we showed that both mRNA and protein expression levels of GPX3 are significantly reduced in cervical cancer tissues compared to normal cervical tissues. In addition, in 5 matched tissue samples, GPX3 protein expression showed a tendency to gradually decrease from normal cervical tissues $(100 \%, 5 / 5)$, primary cervical tissues $(20.0 \%, 1 / 5)$ to lymph node metastases $(0 \%, 0 / 5)$, indicating that the downregulation of GPX3 may also be involved in cervical cancer progression. Furthermore, as in other types of cancer (3), downregulation of GPX3 expression is closely related to lymph node metastasis and prognosis in cervical cancer, indicating the importance of GPX3 expression in cervical cancer. Downregulation of GPX3 induced by promoter methylation has also been observed in gastric carcinoma (26) and Barrett's adenocarcinoma (6). In the present study, we also found that promoter methylation was detected frequently in cervical cancer cell lines and 5-Aza-dC treatment can restore the mRNA expression of GPX3. Hypermethylation of $\mathrm{CpG}$ islands is one of the causes of GPX3 downregulation in cervical cancer.

Sustained level of reactive oxygen species (ROS) generates oxidative stress and results in damages to host cell DNA protein and lipids. ROS-induced DNA can be the most deleterious since it is not easily replaced and, when the DNA repair system does not function properly to prevent the passage of damage to progeny cells, in the most severe case, human disease such as cancer develops (27). From yeast to higher eukaryotes, GPXs and other antioxidant enzymes function to protect cells against oxidative damages. It remains to be examined whether GPX3 functions to suppress cervical cancer promotion in vitro and in vivo.

In conclusion, GPX3 could be a novel predictive molecular biomarker for lymph node metastasis and cancer prognosis in cervical cancer patients.

\section{Acknowledgements}

This research was supported by Basic Science Research Program through the National Research Foundation of Korea (NRF) funded by the Ministry of Science, ICT \& Future Planning (No. 2013R1A2A2A04015894). This work was also partly supported by the Priority Research Centers Program through the NRF funded by the Ministry of Education, Science and Technology (2009-0094027).

\section{References}

1. Lodygin D, Epanchintsev A, Menssen A, Diebold J and Hermeking H: Functional epigenomics identifies genes frequently silenced in prostate cancer. Cancer Res 65: 4218-4227, 2005.

2. Brigelius-Flohé R and Kipp A: Glutathione peroxidases in different stages of carcinogenesis. Biochim Biophys Acta 1790: 1555-1568, 2009

3. Yu YP, Yu G, Tseng G, et al: Glutathione peroxidase 3, deleted or methylated in prostate cancer, suppresses prostate cancer growth and metastasis. Cancer Res 67: 8043-8050, 2007.

4. Mikata R, Yokosuka O, Fukai K, et al: Analysis of genes upregulated by the demethylating agent 5-aza-2'-deoxycytidine in gastric cancer cell lines. Int J Cancer 119: 1616-1622, 2006. 
5. Lee OJ, Schneider-Stock R, McChesney PA, Kuester D, Roessner A, Vieth M, Moskaluk CA and El-Rifai W: Hypermethylation and loss of expression of glutathione peroxidase-3 in Barrett's tumorigenesis. Neoplasia 7: 854-861, 2005.

6. Peng DF, Razvi M, Chen H, et al: DNA hypermethylation regulates the expression of members of the Mu-class glutathione S-transferases and glutathione peroxidases in Barrett's adenocarcinoma. Gut 58: 5-15, 2009.

7. Zhang X1, Yang JJ, Kim YS, Kim KY, Ahn WS and Yang S: An 8-gene signature, including methylated and down-regulated glutathione peroxidase 3, of gastric cancer. Int J Oncol 36: 405-414, 2010

8. Saga Y, Ohwada M, Suzuki M, et al: Glutathione peroxidase 3 is a candidate mechanism of anticancer drug resistance of ovarian clear cell adenocarcinoma. Oncol Rep 20: 1299-1303, 2008.

9. Yang ZL, Yang L, Zou Q, et al: Positive ALDH1A3 and negative GPX3 expressions are biomarkers for poor prognosis of gallbladder cancer. Dis Markers 35: 163-172, 2013.

10. Kaiser MF, Johnson DC, Wu P, et al: Global methylation analysis identifies prognostically important epigenetically inactivated tumour suppressor genes in multiple myeloma. Blood 122: 219-226, 2013

11. El Haddad M, Jean E, Turki A, et al: Glutathione peroxidase 3 , a new retinoid target gene, is crucial for human skeletal muscle precursor cell survival. J Cell Sci 125: 6147-6156, 2012.

12. Barrett CW, Ning W, Chen X, et al: Tumor suppressor function of the plasma glutathione peroxidase gpx 3 in colitis-associated carcinoma. Cancer Res 73: 1245-1255, 2013.

13. Fitzpatrick JM, Schulman C, Zlotta AR and Schröder FH: Prostate cancer: a serious disease suitable for prevention. BJU Int 103: 864-870, 2009.

14. Lu J and Holmgren A: Selenoproteins. J Biol Chem 284: 723-727, 2009

15. Yang X, Deignan JL, Qi H, et al: Validation of candidate causal genes for obesity that affect shared metabolic pathways and networks. Nat Genet 41: 415-423, 2009.

16. Bachtiary B, Boutros PC, Pintilie M, et al: Gene expression profiling in cervical cancer: an exploration of intratumor heterogeneity. Clin Cancer Res 12: 5632-5640, 2006.
17. He Y, Wang Y, Li P, Zhu S, Wang J and Zhang S: Identification of GPX3 epigenetically silenced by $\mathrm{CpG}$ methylation in human esophageal squamous cell carcinoma. Dig Dis Sci 56: 681-688, 2011.

18. Scotto L, Narayan G, Nandula SV, et al: Identification of copy number gain and overexpressed genes on chromosome arm 20q by an integrative genomic approach in cervical cancer: potential role in progression. Genes Chromosomes Cancer 47: 755-765, 2008.

19. Baylin SB and Ohm JE: Epigenetic gene silencing in cancer - a mechanism for early oncogenic pathway addiction? Nat Rev Cancer 6: 107-116, 2006.

20. Esteller M: Epigenetics in cancer. N Engl J Med 358: 1148-1159, 2008.

21. Jones PA and Baylin SB: The fundamental role of epigenetic events in cancer. Nat Rev Genet 3: 415-428, 2002.

22. Christman JK: 5-Azacytidine and 5-aza-2'-deoxycytidine as inhibitors of DNA methylation: mechanistic studies and their implications for cancer therapy. Oncogene 21: 5483-5495, 2002.

23. Brigelius-Flohé R: Glutathione peroxidases and redox-regulated transcription factors. Biol Chem 387: 1329-1335, 2006.

24. Clark LC, Combs GF Jr, Turnbull BW, et al: Effects of selenium supplementation for cancer prevention in patients with carcinoma of the skin. A randomized controlled trial. Nutritional Prevention of Cancer Study Group. JAMA 276: 1957-1963, 1996.

25. Dobosy JR, Roberts JL, Fu VX and Jarrard DF: The expanding role of epigenetics in the development, diagnosis and treatment of prostate cancer and benign prostatic hyperplasia. J Urol 177: 822-831, 2007.

26. Jee CD, Kim MA, Jung EJ, Kim J and Kim WH: Identification of genes epigenetically silenced by CpG methylation in human gastric carcinoma. Eur J Cancer 45: 1282-1293, 2009.

27. Maynard S, Schurman SH, Harboe C, de Souza-Pinto NC and Bohr VA: Base excision repair of oxidative DNA damage and association with cancer and aging. Carcinogenesis 30: 2-10, 2009. 